\title{
Adaptive Fuzzy Controller for a Class of Uncertain Nonlinear Systems
}

\author{
Hugang HAN *
}

\begin{abstract}
In general, the state feedback control gain can be obtained by solving certain linear matrix inequalities (LMIs) when using the Takagi-Sugeno (T-S) fuzzy model to develop a control system. In this paper, the reconstruction error between the real system to be controlled and its T-S fuzzy model, which consists of parameter uncertainties and external disturbance, is considered. As a result, we arrive at an adaptive controller that has two parts: one is obtained by solving certain LMIs (fixed part) and another one is acquired by an adaptive law (variable part). The proposed controller can guarantee the control state to converge and uniformly bounded while maintaining all the signals involved stable. Also, the convergence and boundedness in terms of relaxing the LMIs conservatism are discussed. An inverted pendulum is provided to demonstrate the effectiveness of the proposed adaptive fuzzy controller.
\end{abstract}

Keywords : Linear matrix inequality, adaptive fuzzy controller, conservatism, uniformly boundedness

\section{Introduction}

One of the essential elements of the control problem is the model of the dynamical system to be controlled. In many cases a mathematical model of the system is unavailable or incomplete, or the equations that we believe are adequate to represent the behavior of the system are too complicated for the design purpose. This is the most likely reason why the application of fuzzy set theory to control problems has been the focus of numerous studies. The reason is that the fuzzy set theory provides an alternative to the traditional modeling and design of control systems, where knowledge at the dynamic model of the system in the traditional sense is uncertain and time-varying. In recent years, there have been significant advances in the study of the stability analysis and controller synthesis for the so-called Takagi-Sugeno (T-S), also known as the Takagi-Sugeno-Kang (TSK) fuzzy systems [1] [2], which have been used to represent certain complex nonlinear systems. In the T-S fuzzy model, the local dynamics in different state-space regions are represented by linear models such as $\dot{x}(t)=A_{i} x(t)+B_{i} u$ $(t)$, where $A_{i}, B_{i}$ are certain known matrices with some appropriate dimensions. The overall model of the system is obtained by the fuzzy blending of these

* Department of Management Information System, Prefectural University of Hiroshima local models. The control design is carried out based on the fuzzy model by the so-called parallel distributed compensation (PDC) scheme [3]. For each local linear model, a linear feedback control is designed. The resulting overall controller is again a fuzzy blending of the individual linear controllers. Originally, Tanaka and his colleagues have provided certain conditions that are sufficient for the stability of the T-S fuzzy systems in the sense of Lyapunove [3]-[5]. The conditions for the existence of a common Lyapunov function are obtained by solving linear matrix inequalities (LMIs). Currently, the stability issue about the $\mathrm{T}$ -S fuzzy systems has been extensively studied.

However, only a few results concerning the modeling error, which often is referred to as reconstruction error, between the real system to be controlled and its T-S fuzzy model, are reported [4] [6]-[8]. Basically, the reconstruction error is caused by the uncertainties in the real(nonlinear) system including external disturbance, and the transformation from the real system to the T-S fuzzy model. In [4], they have concluded some LMI conditions to confine the influence of the reconstruction error to a certain region. More popularly, the reconstruction error is treated by involving some norm-bounded matrices with certain structure properties in each local T-S fuzzy model 
such as $\dot{x}(t)=\left(A_{i}+\Delta A_{i}\right) x(t)+\left(B_{i}+\Delta B_{i}\right) u(t)$, where $\Delta A_{i}, \Delta B_{i}$ represent the norm-bounded matrices [6] [8], consequently, the LMI technique can be applied to obtain the fuzzy controller. However, the norm-bounded matrices are not easy to be estimated since the mathematical model of the real system is unavailable in most cases, and often being set large to safely cover the reconstruction error. At the same time, the large norm-bounded matrices inserted in each local T-S fuzzy model may lead the final LMIs to being conservative. In [7], Zhang and his colleagues have firstly considered an external disturbance term in addition to the norm-bounded matrices in each local T-S fuzzy model such as $\dot{x}(t)=\left(A_{i}+\right.$ $\left.\Delta A_{i}\right) x(t)+B_{i} u(t)+D_{i}$, where $D_{i}$ denotes the external disturbance, and proposed a robust fuzzy controller that can deal with both $\Delta A_{i}$ and $D_{i}$. However, the results may be limited as we expected since they did not consider uncertainties in the gain matrix $B_{i}$.

In this paper, in order to effectively deal with the reconstruction error, each local T-S fuzzy model is formed of $\dot{x}(t)=\left(A_{i}+\Delta A_{i}\right) x(t)+\left(B_{i}+\Delta B_{i}\right) u(t)$ $+D_{i}$, and an adaptive controller, which has two parts: one is obtained by solving certain LMIs (fixed part) and the another one is acquired by an adaptive law (variable part), is proposed. The proposed controller can guarantee the control state to converge and uniformly bounded while maintaining all the signals involved stable. Also, the convergence and boundedness in terms of relaxing the LMIs conservatism are discussed. An inverted pendulum is provided to demonstrate the effectiveness of the proposed adaptive fuzzy controller.

\section{Problem Statement}

\subsection{System and its T-S Fuzzy Model}

Consider the following uncertain nonlinear system:

$$
\begin{aligned}
\dot{x}(t)= & f(x, t)+\Delta f(x, t, \xi) \\
& +(g(x, t)+\Delta g(x, t, \xi)) u(t)+\psi(x, t, \xi),
\end{aligned}
$$

where $x(t) \in \mathcal{R}^{n}$ is the vector of state variables; $u(t)$ $\in \mathcal{R}^{m}$, the vector of control inputs; $\xi$, a vector of uncertain parameters that is restricted to a prescribed bounded set $\Xi ; f$ and $g$, nonlinear functions of $(x, t)$, continuously differentiable with respect to $(x, t)$;
$\Delta f$ and $\Delta g$, the model uncertainties; and $\psi$, the external disturbance.

With the uncertainties and disturbance in consideration, such a system can be expressed in the terms of $r$ fuzzy rules in the T-S fuzzy model as follows.

Plant Rule $i$ :

IF $\theta_{1}(t)$ is $F_{1}^{i}$ AND $\cdots$ AND $\theta_{p}(t)$ is $F_{p}^{i}$, THEN

$$
\begin{aligned}
\dot{x}(t) & =\left(A_{i}+\Delta A_{i}(x, t, \xi)\right) x(t) \\
& +\left(B_{i}+\Delta B_{i}(x, t, \xi)\right) u(t)+D_{i}(x, t, \xi),
\end{aligned}
$$

where constant matrices $A_{i}$ and $B_{i}$ are of appropriate dimensions, $\Delta A_{i}, \Delta B_{i}$ represent the uncertainties in system matrices, and $D_{i}$ stands for external disturbance. The overall fuzzy model is of the following form accordingly:

$$
\begin{aligned}
\dot{x}(t)= & \sum_{i=1}^{r} \alpha_{i}(t)\left[\left(A_{i}+\Delta A_{i}(x, t, \xi)\right) x(t)\right. \\
& \left.+\left(B_{i}+\Delta B_{i}(x, t, \xi)\right) u(t)+D_{i}(x, t, \xi)\right],
\end{aligned}
$$

where

$$
\begin{gathered}
\alpha_{i}(t)=\frac{w_{i}(t)}{\sum_{i=1}^{r} w_{i}(t)} \geq 0, \quad \sum_{i=1}^{r} \alpha_{i}(t)=1, \\
w_{i}(t)=\prod_{j=1}^{p} \mu_{j}^{i}\left(\theta_{j}(t)\right) .
\end{gathered}
$$

\subsection{Matching Conditions}

In this paper, the uncertainties $\Delta A_{i}$ and $\Delta B_{i}$ are assumed to be of the following structure [6] [9]:

$$
\left[\Delta A_{i}(x, t, \xi) \quad \Delta B_{i}(x, t, \xi)\right]=M_{i} \Theta(x, t, \xi)\left[N_{1 i} \quad N_{2 i}\right],
$$

where $M_{i}, N_{1 i}$ and $N_{2 i}$ are known constant matrices with compatible dimensions, and $\Theta(x, t, \xi)$ is unknown nonlinear time-varying matrix function satisfying

$\|\Theta(x, t, \xi)\| \leq 1$,

where the elements of $\Theta(x, t, \xi)$ are assumed to be Lebesgue measurable, and $\|\cdot\|$ denotes the Euclidean norm of a matrix (or vector) in this paper. This type of uncertainty is an effective representation of some nonlinear uncertainties. As mentioned in [9], there are several reasons for assuming that the system un- 
certainty has the structure given in (3). First, a linear interconnection of a nominal plant with the uncertainty $\Theta(x, t, \xi)$ leads to a structure of the form (3). In addition, there are many physical systems in which the uncertainty can be modeled in this manner, e.g., systems satisfying "matching conditions".

As to disturbance, all the external disturbances $D_{i}$ $(x, t, \xi)(\mathrm{i}=1,2, \ldots, r)$ are norm-bounded and satisfy the following matching condition:

$$
\begin{aligned}
& D_{i}(x, t, \xi)=B_{i} d_{i}(x, t, \xi) \\
& \left\|d_{i}(x, t, \xi)\right\| \leq d_{i}^{*},
\end{aligned}
$$

where the upper bounds $d_{i}^{*}$ are not necessary to be known in this paper. As shown latter on, its estimate $\hat{d}_{i}$ will be given based on an adaptive law.

To proceed with our development, we state an assumption stipulated on the plant:

$\bar{b} \leq 2 \underline{b}$,

where $\bar{b}=\max \left\{\left\|B_{i}\right\|\right\}_{i=1}^{r}, \underline{b}=\min \left\{\left\|B_{i}\right\|\right\}_{i=1}^{r}$.

In case the matching condition (7) does not hold, we can modify $B_{i}$ 's in certain local linear models, since the modified parts are able to be covered by $d_{i}$ 's in (5), which is estimated by an adaptive law in this paper.

From here, unless confusion arises arguments such as $t$ and $\xi$ will be omitted just for notational convenience.

\subsection{Control Objective}

The control objective is to design the following PDC controller [3] [4] for the fuzzy model (2).

\section{Control Rule $i$ :}

IF $\theta_{1}(t)$ is $F_{1}^{i}$ AND $\cdots$ AND $\theta_{p}(t)$ is $F_{p}^{i}$,

THEN $u(t)=K_{i} x(t)$,

where $K_{i} \in \mathcal{R}^{n}(i=1,2, \ldots, r)$ are the controller gain matrices to be designed. The overall state feedback control law is finally represented as

$u=\sum_{i=1}^{r} \alpha_{i} K_{i} x$

With this fuzzy controller, the closed-loop system is written as

$$
\begin{aligned}
\dot{x}=\sum_{i=1}^{r} & \sum_{j=1}^{r} \alpha_{i} \alpha_{j}\left\{\left(A_{i}+B_{i} K_{j}\right.\right. \\
& \left.\left.+\Delta A_{i}+\Delta B_{i} K_{j}\right) x+D_{i}\right\}
\end{aligned}
$$

The aim of this paper is to design $r$ local linear state feedback law (8) for the T-S fuzzy model (2) such that the solutions of (10) are uniformly bounded while maintaining all signals involved stable.

\section{Main Results}

\subsection{Controller Design}

In order to develop our fuzzy control system, the following preliminary lemma is required [10].

Lemma 1 Let $Q \in \mathcal{R}^{n \times n}$ be any matrix, we have, for any $k>0$ any symmetric positive matrix $T>0$, that

$2 x^{T} Q y \leq k x^{T} Q T^{-1} Q^{T} x+\frac{1}{k} y^{T} T y$

for $\forall x, y \in \mathcal{R}^{n}$.

Now, for given feedback control gain $K_{i}$ in (8), we give the following theorem for the system stability conditions of (10).

Theorem 1 For a given fuzzy model (2) with uncertainties and disturbance subject to the matching conditions (3)-(7), if there exist certain symmetric matrices $P>0, T_{1 i}>0, T_{2 i}>0(i=1,2, \ldots, r)$, and matrices $\mathcal{K}_{j}(j=1,2, \ldots, r)$, such that the following LMIs:

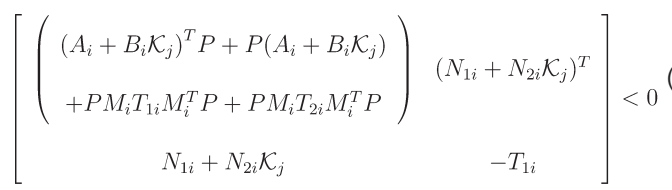

hold, then the feedback control gain $K_{i}$ in (8):

$K_{i}=\mathcal{K}_{i}+\Phi_{i}$,

where

$\Phi_{i}=-\delta \hat{d}_{i}^{2} B_{i}^{T} P$

$\dot{\hat{d}}_{i}(t)=-\alpha_{i} \beta_{i} \gamma_{i} \hat{d}_{i}(t)+\alpha_{i} \gamma_{i}(\bar{b}\|P x\|)^{1 / 2}$

$\delta \leq \frac{2 \underline{b}-\bar{b}}{\hat{d}_{i}^{2} \lambda_{\max } \bar{b}}$

$\lambda_{\max }=\left\{\lambda\left(N_{2 i}^{T} T_{2 i}^{-1} N_{2 i}\right)\right\}_{i=1}^{r}$ which $i$ s the maximum eigenvalue (scalar) of square matrices $N_{2 i}^{T} T_{2 i}^{-1} N_{2 i}$ 
$(i=1,2, \ldots, r)$, and $\beta_{i}>0, \gamma_{i}>0$ are design parameters, will make the solutions of the closed-loop system (10) uniformly bounded while maintaining all signals involved stable. At the same time, the solutions exponentially converge at rate $\frac{\eta}{2}$ to a ball centered at the equilibrium point with radius $\tau$, where $\eta$ is given in (37), (34), (35), (24), (26), and $\tau$ is given in (40) and (32).

Proof: Define a Lyapunov function as follows.

$V(z(t))=x^{T} P x+\sum_{i=1}^{r} \gamma_{i}^{-1} \tilde{d}_{i}^{2}$,

where $z=\left[x, \tilde{d}_{1}, \tilde{d}_{2}, \ldots, \tilde{d}_{r}\right]^{T}, \tilde{d}_{i}=d_{i}^{*}-\hat{d}_{i}$. The time derivative of $V(z)$ on any trajectory of the closed-loop system is

$$
\begin{aligned}
\dot{V}= & 2 \dot{x}^{T} P x-2 \sum_{i=1}^{r} \gamma_{i}^{-1} \tilde{d}_{i} \dot{d}_{i} \\
= & 2 \sum_{i=1}^{r} \sum_{j=1}^{r} \alpha_{i} \alpha_{j}\left\{x ^ { T } \left(A_{i}+\Delta A_{i}+B_{i} K_{j}\right.\right. \\
& \left.\left.+\Delta B_{i} K_{j}\right)^{T}+D_{i}^{T}\right\} P x-2 \sum_{i=1}^{r} \gamma_{i}^{-1} \tilde{d}_{i} \dot{d}_{i} \\
= & \sum_{i=1}^{r} \sum_{j=1}^{r} \alpha_{i} \alpha_{j} x^{T}\left\{\left(A_{i}+B_{i} \mathcal{K}_{j}\right)^{T} P+P\left(A_{i}+B_{i} \mathcal{K}_{j}\right)\right\} x \\
& +2 \sum_{i=1}^{r} \sum_{j=1}^{r} \alpha_{i} \alpha_{j}\left\{x^{T}\left(\Delta A_{i}+\Delta B_{i} \mathcal{K}_{j}+\Delta B_{i} \Phi_{j}+B_{i} \Phi_{j}\right)^{T}+D_{i}^{T}\right\} P x \\
& -2 \sum_{i=1}^{r} \gamma_{i}^{-1} \tilde{d}_{i} \hat{d}_{i} .
\end{aligned}
$$

According to the matching condition (3), (4) and Lemma 1, we have

$$
\begin{aligned}
& 2 x^{T}\left(\Delta A_{i}+\Delta B_{i} \mathcal{K}_{j}\right)^{T} P x \\
= & 2 x^{T}\left(M_{i} \Theta\left(N_{1 i}+N_{2 i} \mathcal{K}_{j}\right)\right)^{T} P x \\
= & 2 x^{T}\left(N_{1 i}+N_{2 i} \mathcal{K}_{j}\right)^{T} \Theta^{T} M_{i}^{T} P x \\
\leq & x^{T}\left(N_{1 i}+N_{2 i} \mathcal{K}_{j}\right)^{T} \Theta^{T} T_{1 i}^{-1} \Theta\left(N_{1 i}+N_{2 i} \mathcal{K}_{j}\right) x \\
& +x^{T} P M_{i} T_{1 i} M_{i}^{T} P x \\
\leq & x^{T}\left(N_{1 i}+N_{2 i} \mathcal{K}_{j}\right)^{T} T_{1 i}^{-1}\left(N_{1 i}+N_{2 i} \mathcal{K}_{j}\right) x \\
& +x^{T} P M_{i} T_{1 i} M_{i}^{T} P x .
\end{aligned}
$$

By the same token, it follows that

$2 x^{T}\left(\Delta B_{i} \Phi_{j}\right)^{T} P x \leq x^{T} \Phi_{j}^{T} N_{2 i}^{T} T_{2 i}^{-1} N_{2 i} \Phi_{j} x+x^{T} P M_{i} T_{2 i} M_{i}^{T} P x$.

Substituting (19) and (20) in (18), we have

$$
\dot{V} \leq V_{1}+V_{2},
$$

where

$$
\begin{aligned}
V_{1}= & x^{T} \sum_{i=1}^{r} \sum_{j=1}^{r} \alpha_{i} \alpha_{j}\left\{\left(A_{i}+B_{i} \mathcal{K}_{j}\right)^{T} P+P\left(A_{i}+B_{i} \mathcal{K}_{j}\right)\right. \\
& +P M_{i} T_{1 i} M_{i}^{T} P+P M_{i} T_{2 i} M_{i}^{T} P \\
& \left.+\left(N_{1 i}+N_{2 i} \mathcal{K}_{j}\right)^{T} T_{1 i}^{-1}\left(N_{1 i}+N_{2 i} \mathcal{K}_{j}\right)\right\} x \\
V_{2}= & \sum_{i=1}^{r} \sum_{j=1}^{r} \alpha_{i} \alpha_{j}\left[x ^ { T } \left\{2\left(B_{i} \Phi_{j}\right)^{T} P\right.\right. \\
& \left.\left.+\Phi_{j}^{T} N_{2 i}^{T} T_{2 i}^{-1} N_{2 i} \Phi_{j}\right\} x+2 D_{i}^{T} P x\right]-\sum_{i=1}^{r} 2 \gamma_{i}^{-1} \tilde{d}_{i} \dot{\hat{d}}_{i} .
\end{aligned}
$$

We first consider $V_{1}$. Let

$$
\begin{aligned}
-S_{i j}= & \left(A_{i}+B_{i} \mathcal{K}_{j}\right)^{T} P+P\left(A_{i}+B_{i} \mathcal{K}_{j}\right) \\
& +P M_{i} T_{1 i} M_{i}^{T} P+P M_{i} T_{2 i} M_{i}^{T} P \\
& +\left(N_{1 i}+N_{2 i} \mathcal{K}_{j}\right)^{T} T_{1 i}^{-1}\left(N_{1 i}+N_{2 i} \mathcal{K}_{j}\right)
\end{aligned}
$$

Then, by the Schur complement LMIs (12) are equivalent to $S_{i j}>0$. Therefore, we have

$V_{1}=-x^{T} \mathcal{S} x<0$,

where

$$
\mathcal{S}=\sum_{i=1}^{r} \sum_{j=1}^{r} \alpha_{i} \alpha_{j} S_{i j}>0 .
$$

Next, let us consider $V_{2}$. Substituting matching condition (5), variable control gain (14) and adaptive law (15) in (23), it follows that

$$
\begin{aligned}
& V_{2} \leq \sum_{i=1}^{r} \sum_{j=1}^{r} \alpha_{i} \alpha_{j}\left\{-2 \delta \hat{d}_{j}^{2} x^{T} P B_{j} B_{i}^{T} P x+\delta^{2} \hat{d}_{j}^{4} \lambda_{\max } \bar{b}\|P x\|+2 d_{i}^{*}(\bar{b}\|P x\|)^{\frac{1}{2}}\right\} \\
& +\sum_{i=1}^{r} \alpha_{i}\left\{2 \beta_{i} \tilde{d}_{i} \hat{d}_{i}-2 \tilde{d}_{i}(\bar{b}\|P x\|)^{\frac{1}{2}}\right\} \\
& \left.\leq \sum_{i=1}^{r} \sum_{j=1}^{r} \alpha_{i} \alpha_{j}\left\{-2 \delta \hat{d}_{j}^{2} \underline{b}\|P x\|+\delta^{2} \hat{d}_{j}^{4} \lambda_{\max } \bar{b}\|P x\|+2 d_{i}^{*} \bar{b}\|P x\|\right)^{\frac{1}{2}}\right\} \\
& +\sum_{i=1}^{r} \alpha_{i}\left\{2 \beta_{i} \tilde{d}_{i} \hat{d}_{i}-2 \tilde{d}_{i}(\bar{b}\|P x\|)^{\frac{1}{2}}\right\} \\
& =\sum_{i=1}^{r} \alpha_{i}\left\{-2 \delta \hat{d}_{i}^{2} \underline{b}\|P x\|+\delta^{2} \hat{d}_{i}^{4} \lambda_{\max } \bar{b}\|P x\|+2 d_{i}^{*}(\bar{b}\|P x\|)^{\frac{1}{2}}\right\} \\
& +\sum_{i=1}^{r} \alpha_{i}\left\{2 \beta_{i} \tilde{d}_{i} \hat{d}_{i}-2 \tilde{d}_{i}(\bar{b}\|P x\|)^{\frac{1}{2}}\right\} \\
& =\sum_{i=1}^{r} \alpha_{i}\left\{-2 \delta \hat{d}_{i}^{2} \underline{b}\|P x\|+\delta^{2} \hat{d}_{i}^{4} \lambda_{\max } \bar{b}\|P x\|+2 d_{i}^{*}(\bar{b}\|P x\|)^{\frac{1}{2}}\right. \\
& \left.+2 \beta_{i} \tilde{d}_{i} \hat{d}_{i}-2 \tilde{d}_{i}(\bar{b}\|P x\|)^{\frac{1}{2}}\right\} \\
& =\sum_{i=1}^{r} \alpha_{i}\left\{-2 \delta \hat{d}_{i}^{2} \underline{b}\|P x\|+\delta^{2} \hat{d}_{i}^{4} \lambda_{\max } \bar{b}\|P x\|+2 \hat{d}_{i}(\bar{b}\|P x\|)^{\frac{1}{2}}+2 \beta_{i} \tilde{d}_{i} \hat{d}_{i}\right\},
\end{aligned}
$$

where the facts that $\sum_{i=1}^{r} \alpha_{i}=\sum_{j=1}^{r} \alpha_{j}=1, \tilde{d}_{i}=d_{i}^{*}-$ 
$\hat{d}_{i}$ are used. Let

$$
\Delta b=\bar{b}-\underline{b} .
$$

Substituting $\underline{b}=\bar{b}-\Delta b$ in (27), it becomes

$$
\begin{aligned}
V_{2} \leq & \sum_{i=1}^{r} \alpha_{i}\left\{-2 \delta \hat{d}_{i}^{2} \bar{b}\|P x\|+2 \hat{d}_{i}(\bar{b}\|P x\|)^{\frac{1}{2}}\right. \\
& \left.+2 \delta \hat{d}_{i}^{2} \Delta b\|P x\|+\delta^{2} \hat{d}_{i}^{4} \lambda_{\max } \bar{b}\|P x\|+2 \beta_{i} \tilde{d}_{i} \hat{d}_{i}\right\} .
\end{aligned}
$$

Since the facts that

$$
\begin{aligned}
& -\delta \hat{d}_{i}^{2} \bar{b}\|P x\|+2 \hat{d}_{i}(\bar{b}\|P x\|)^{\frac{1}{2}} \\
& =-\left\{\hat{d}_{i}(\delta \bar{b}\|P x\|)^{\frac{1}{2}}-\delta^{-\frac{1}{2}}\right\}^{2}+\frac{1}{\delta} \leq \frac{1}{\delta} \\
& 2 \beta_{i} \tilde{d}_{i} \hat{d}_{i}=2 \beta_{i} \tilde{d}_{i} d_{i}^{*}-2 \beta_{i} \tilde{d}_{i}^{2} \\
& =-\beta_{i} \tilde{d}_{i}^{2}-\beta_{i}\left(\tilde{d}_{i}^{2}-2 \tilde{d}_{i} d_{i}^{*}+d_{i}^{* 2}\right)+\beta_{i} d_{i}^{* 2} \\
& \leq-\beta_{i} \tilde{d}_{i}^{2}+\beta_{i} d_{i}^{* 2}
\end{aligned}
$$

hold, it yields that

$$
\begin{aligned}
V_{2} \leq & \sum_{i=1}^{r} \alpha_{i}\left\{\frac{1}{\delta}-\delta \hat{d}_{i}^{2} \bar{b}\|P x\|-\beta_{i} \tilde{d}_{i}^{2}+\beta_{i} d_{i}^{* 2}\right. \\
& \left.+2 \delta \hat{d}_{i}^{2} \Delta b\|P x\|+\delta^{2} \hat{d}_{i}^{4} \lambda_{\max } \bar{b}\|P x\|\right\} \\
= & \sum_{i=1}^{r} \alpha_{i}\left\{\frac{1}{\delta}-\beta_{i} \tilde{d}_{i}^{2}+\beta_{i} d_{i}^{* 2}\right. \\
& \left.-\delta \hat{d}_{i}^{2}\|P x\|\left(\bar{b}-2 \Delta b-\delta \hat{d}_{i}^{2} \lambda_{\max } \bar{b}\right)\right\} .
\end{aligned}
$$

Now, let us pay attention to the last term in the left side of the above inequality. From (16) and (28), it is clear that $\delta \hat{d}_{i}^{2}\left\|P_{x}\right\|\left(\bar{b}-2 \Delta b-\delta \hat{d}_{i}^{2} \lambda_{\max } \bar{b}\right) \geq 0$. Thus,

$$
\begin{aligned}
V_{2} & \leq \sum_{i=1}^{r} \alpha_{i}\left(-\beta_{i} \tilde{d}_{i}^{2}+\frac{1}{\delta}+\beta_{i} d_{i}^{* 2}\right) \\
& =-\sum_{i=1}^{r} \alpha_{i} \beta_{i} \tilde{d}_{i}^{2}+\varepsilon
\end{aligned}
$$

where

$$
\varepsilon=\sum_{i=1}^{r} \alpha_{i}\left(\frac{1}{\delta}+\beta_{i} d_{i}^{* 2}\right) .
$$

Substituting (25) and (31) in (21), it follows that

$$
\begin{aligned}
\dot{V} & \leq-x^{T} \mathcal{S} x-\sum_{i=1}^{r} \alpha_{i} \beta_{i} \tilde{d}_{i}^{2}+\varepsilon \\
& \leq-\lambda_{\min }(\mathcal{S}) \lambda_{\max }^{-1}(P) x^{T} P x-\sum_{i=1}^{r} \alpha_{i} \beta_{i} \gamma_{i} \gamma_{i}^{-1} \tilde{d}_{i}^{2}+\varepsilon .
\end{aligned}
$$

Let

$$
\begin{aligned}
& \eta_{1}=\lambda_{\min }(\mathcal{S}) \lambda_{\max }^{-1}(P) \\
& \eta_{2}=\min \left\{\alpha_{1} \beta_{1} \gamma_{1}, \alpha_{2} \beta_{2} \gamma_{2}, \ldots, \alpha_{r} \beta_{r} \gamma_{r}\right\} .
\end{aligned}
$$

Then,

$$
\begin{aligned}
& \dot{V} \leq-\eta_{1} x^{T} P x-\sum_{i=1}^{r} \eta_{2} \gamma_{i}^{-1} \tilde{d}_{i}^{2}+\varepsilon \\
& \leq-\eta\left(x^{T} P x+\sum_{i=1}^{r} \gamma_{i}^{-1} \tilde{d}_{i}^{2}\right)+\varepsilon \\
& =-\eta V+\varepsilon,
\end{aligned}
$$

where

$$
\eta=\min \left\{\eta_{1}, \eta_{2}\right\}
$$

From (36) we have

$$
V \leq \frac{\varepsilon}{\eta}+\left(V(0)-\frac{\varepsilon}{\eta}\right) e^{-\eta t},
$$

where $V(0)=\left.V(z(t))\right|_{t=0}$. Since that $\eta>0$, the second block on the right side of (38) tends to 0 when $t$ $\rightarrow \infty$. Therefore, we can get that there exists $\mathcal{T}$ such that for $t \geq \mathcal{T}$ it satisfies

$\|x\| \leq \tau$

where

$$
\tau=\frac{1}{\lambda_{\min }(P)}\left(\frac{\varepsilon}{\eta}\right)^{\frac{1}{2}} .
$$

This completes the proof.

The stability conditions presented in Theorem 1 can be used to obtain an LMI-based design for the feedback gain $K_{i}$ in (8). If we define $Q=P^{-1}$, the following theorem comes.

Theorem 2 For a given fuzzy model (2) with uncertainties and disturbance subject to the matching conditions (3)-(7), if there exist certain symmetric matrices $Q>0, T_{1 i}>0, T_{2 i}>0(i=1,2, \ldots, r)$, and matrices $G_{j}(j=1,2, \ldots, r)$ such that the following LMIs:

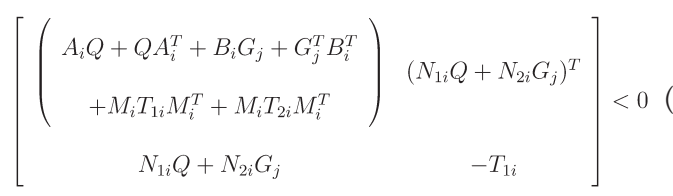

hold, then the feedback control gain Ki in (8):

$K_{i}=\mathcal{K}_{i}+\Phi_{i}$, 
where

$\mathcal{K}_{j}=G_{j} Q^{-1}$

$\Phi_{i}=-\delta \hat{d}_{i}^{2} B_{i}^{T} Q^{-1}$

$\dot{\hat{d}}_{i}(t)=-\alpha_{i} \beta_{i} \gamma_{i} \hat{d}_{i}(t)+\alpha_{i} \gamma_{i}\left(\bar{b}\left\|Q^{-1} x\right\|\right)^{1 / 2}$

$\delta \leq \frac{2 \underline{b}-\bar{b}}{\hat{d}_{i}^{2} \lambda_{\max } \bar{b}}$

$\lambda_{\max }=\left\{\lambda\left(N_{2 i}^{T} T_{2 i}^{-1} N_{2 i}\right)\right\}_{i=1}^{r}$ which is the maximum eigenvalue (scalar) of square matrices $N_{2 i}^{T} T_{2 i}^{-1} N_{2 i}$ $(i=1,2, \ldots, r)$, and $\beta_{i}>0, \gamma_{i}>0$ are design $p a-$ rameters, will make the solutions of the closed-loop system (10) uniformly bounded while maintaining all signals involved stable. At the same time, the solutions exponentially converge at rate $\frac{\eta}{2}$ to a ball centered at the equilibrium point with radius $\tau$, where $\eta$ and $\tau$ are provided as follows.

$$
\begin{aligned}
\eta= & \min \left\{\lambda_{\min }(\mathcal{S}) \lambda_{\max }^{-1}\left(Q^{-1}\right), \beta_{1} \gamma_{1}, \beta_{2} \gamma_{2}, \ldots, \beta_{r} \gamma_{r}\right\}(47) \\
\mathcal{S}= & -\sum_{i=1}^{r} \sum_{j=1}^{r} \alpha_{i} \alpha_{j}\left\{A_{i} Q+Q A_{i}^{T}+B_{i} G_{j}+G_{j}^{T} B_{i}^{T}\right. \\
& +M_{i} T_{1 i} M_{i}^{T}+M_{i} T_{2 i} M_{i}^{T} \\
& \left.+\left(N_{1 i} Q+N_{2 i} G_{j}\right)^{T} T_{1 i}^{-1}\left(N_{1 i} Q+N_{2 i} G_{j}\right)\right\} \\
\tau= & \frac{1}{\lambda_{\min }\left(Q^{-1}\right)}\left(\frac{\varepsilon}{\eta}\right)^{\frac{1}{2}} \\
\varepsilon= & \sum_{i=1}^{r} \alpha_{i}\left(\frac{1}{\delta}+\beta_{i} d_{i}^{* 2}\right) .
\end{aligned}
$$

\subsection{Relaxing Conservatism}

In theorems 1 and 2, it is desirable to have the largest $\eta$ and the smallest $\tau$ so that the system state $x$ can shrink most quickly in a ball centered at the equilibrium point with radius $\tau$. Therefore, the results obtained above are basically conservative since they took no account of the matter.

On the other hand, to our best knowledge, there is not an analytical approach to solve the optimization problem with respect to $\eta$ and $\tau$. Therefore, the only way left to us is that we do our best to increase $\eta$ and reduce $\tau$. From (34), (35) and (37), we observe that a bigger $\beta_{i}, \gamma_{i}, \mathcal{S}$, and a smaller $P$ can help make a larger $\eta$. At the same time, from (32) and (40) we know that a bigger $\beta_{i}$ leads to a bigger $\varepsilon$, further to a bigger $\tau$ as well as a smaller $P$. Therefore, in order to make $\eta$ big, we can do something on $\gamma_{i}$, and $\mathcal{S}$ as follows.

- Choosing a bigger $\gamma_{i}$. In the adaptive law (15), since $\gamma_{i}$ is a free design parameter, basically it can be chosen arbitrarily large. However, there is a limit that a large $\gamma_{i}$ can lead to a big $\eta_{1}$, further to a big $\eta$ if $\eta_{1}<\eta_{2}$ in (37).

- Making $-\mathcal{S}_{i j}$ minimum. It is clear that the smallest$\mathcal{S}_{i j}$ is equivalent to the largest $\mathcal{S}_{i j}$, further to the largest $\mathcal{S}$.

Next, let us consider the problem of making $\tau$ small. As mentioned previously, since making a bigger $P$ is in conflict with increasing $\eta$, therefore, how to reduce $\varepsilon$ is a key point except for increasing $\eta$ in (40). From (32), it follows that a large $\delta$ and small $\beta_{i}$ lead to a small $\varepsilon$. However, choosing a small $\beta_{i}$ is also in conflict with making $\eta_{2}$ big, therefore, the only way left is trying to make $\delta$ as large as possible. From (46), we arrive at the following point.

- Making $T_{2 i}^{-1}$ minimum. It is is equivalent to making $\lambda_{\max }$ minimum, which leads to a smaller $\lambda$.

Summarizing above, in order to increase $\eta$ and reduce $\tau$, making both $-\mathcal{S}_{i j}$ and $T_{2 i}^{-1}$ minimum are necessary, which can be transformed in

$-\mathcal{S}_{i j}+I_{M} T_{2 i}^{-1} I_{M}^{T} \rightarrow \min$,

where $I_{M}=\operatorname{block} \operatorname{diag}(I, 0)$ is a block triangular matrix with compatible dimensions. Finally, by the Schur complement, we formulate the requirement of (51) as the following generalized eigenvalue minimization under LMI constraints:

$$
\text { s.t. }\left[\begin{array}{cc}
-\mathcal{S}_{i j} & I_{M} \\
I_{M}^{T} & -T_{2 i}
\end{array}\right]<\lambda \mathcal{M}
$$

where $\mathcal{M}>0$ is an arbitrarily given matrix with compatible dimension. Therefore, the following theorem yields in consideration of relaxing conservatism in Theorem 2.

Theorem 3 For a given fuzzy model (2) with uncertainties and disturbance subject to the matching conditions (3)-(7), if there exist certain symmetric matrix $Q>0, T_{1 i}>0$, 


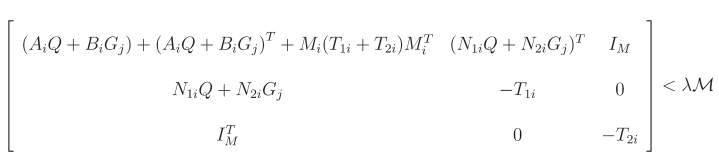

$T_{2 i}>0(i=1,2, \ldots, r)$, and matrices $G_{j}(j=1,2, \ldots$, $r)$ such that the following LMIs:

$$
\text { s.t. }\left\{\begin{array}{l}
\min \lambda \\
L M I(41) \\
L M I(53)
\end{array}\right.
$$

hold, then the feedback control gain $K_{i}$ obtained in (42)-(46) will make the solutions of the closed-loop system (10) uniformly bounded while maintaining all signals involved stable. Also, the solutions exponentially converge at a bigger rate $\frac{\eta}{2}$ to a ball centered at the equilibrium point with a smaller radius $\tau$.

\section{Simulation}

Consider the problem of balancing and swing-up of an inverted pendulum on a cart. The equations of motion for the pendulum are

$$
\begin{aligned}
& \dot{x}_{1}=x_{2} \\
& \dot{x}_{2}=\frac{g \sin \left(x_{1}\right)-\frac{a m l x_{2}^{2} \sin \left(2 x_{1}\right)}{2}-\mu a \cos \left(x_{1}\right) u}{\frac{4 l}{3}-a m l \cos ^{2}\left(x_{1}\right)}+\psi(t),
\end{aligned}
$$

where $x_{1}$ denotes the angle of the pendulum from the vertical; $x_{2}$, the angular velocity; $g=9.8 \mathrm{~m} / \mathrm{s}^{2}$, the gravity constant; $m$, the mass of the pendulum; $M$, the mass of the cart (in kilo-Newton); $\psi(t)$, the external disturbance that is assumed to be a square wave with amplitude \pm 5.0 and period $2 \pi ; a=\frac{1}{m+M}$ and $\mu=1000$. We choose $m=2.0 \mathrm{~kg}, M=8.0 \mathrm{~kg}, l=0.5 \mathrm{~m}$ in this simulation.

The control objective is to balance the inverted pendulum. Here, we follow [6] to approximate the system by the following two-rule fuzzy model:

Rule 1 : IF $x_{1}$ is $\widetilde{0}$ THEN

$$
\dot{x}=\left(A_{1}+\Delta A_{1}\right) x+\left(B_{1}+\Delta B_{1}\right) u+D_{1}
$$

Rule $2:$ IF $x_{1}$ is $\pm \widetilde{\pi / 2}$ THEN

$$
\dot{x}=\left(A_{2}+\Delta A_{2}\right) x+\left(B_{2}+\Delta B_{2}\right) u+D_{2},
$$

where

$$
A_{1}=\left[\begin{array}{cc}
0 & 1 \\
\frac{g}{\frac{4 l}{3}-a m l \beta^{2}} & 0
\end{array}\right], \quad B_{1}=\left[\begin{array}{c}
0 \\
\frac{-\mu a}{\frac{4 l}{3}-a m l}
\end{array}\right],
$$

$A_{2}=\left[\begin{array}{cc}0 & 1 \\ \frac{g}{\pi\left(\frac{4 l}{3}-a m l \beta^{2}\right)} & 0\end{array}\right], \quad B_{2}=\left[\begin{array}{c}0 \\ \frac{-\mu a}{\frac{4 l}{3}-a m l \beta^{2}}\end{array}\right]$,

$\beta=\cos \left(86^{\circ}\right) ; \Delta A_{i}, \Delta B_{i}(i=1,2)$ are uncertainties that describe modeling errors; $D_{i}$, the counterpart of the external disturbance $\psi$ in (56). The membership functions for fuzzy sets $\tilde{0}$, and $\widetilde{\pi / 2}$ are chosen as follows.

$$
\mu_{\tilde{0}}\left(x_{1}\right)=\left\{\begin{array}{cl}
\cos \left(x_{1}\right), & -\frac{\pi}{2} \leq x_{1} \leq \frac{\pi}{2} \\
0, & \text { otherwise }
\end{array}\right.
$$

$\mu_{\pi / 2}\left(x_{1}\right)=\left\{\begin{array}{cc}1-\cos \left(x_{1}\right), & -\frac{\pi}{2} \leq x_{1} \leq \frac{\pi}{2} \\ 0, & \text { otherwise }\end{array}\right.$

which are shown in Fig.1. Since the fact that $\left\|B_{1}\right\|=$ 176.4706 and $\left\|B_{2}\right\|=150.1096$, which implies that $\bar{b}=$ 176.4706 and $\underline{b}=150.1096$, it is clear that the fuzzy model satisfies the assumption, $\bar{b} \leq 2 \underline{b}$, shown in (7). In what follows, we consider the modeling errors $\Delta A_{i}$ and $\Delta B_{i}$, which are assumed to satisfy the matching condition as shown in (3) and (4):

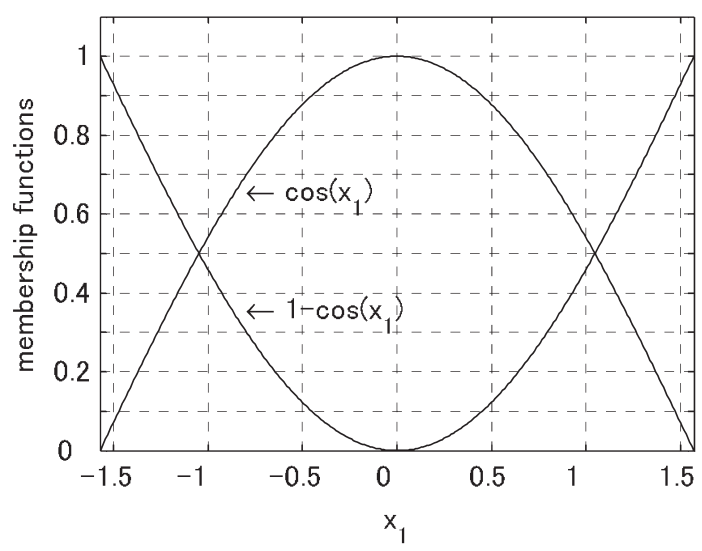

Fig.1 Membership functions of $\mu_{\tilde{0}}\left(x_{1}\right)$ and $\mu_{\pi / 2}\left(x_{1}\right)$ 


$$
\begin{aligned}
& \Delta A_{1}=M_{1} \Theta N_{11}, \quad \Delta B_{1}=M_{1} \Theta N_{12}, \\
& \Delta A_{2}=M_{2} \Theta N_{21}, \quad \Delta B_{2}=M_{2} \Theta N_{22},
\end{aligned}
$$

where $\Theta=\operatorname{diag}\left(\theta_{1}, \theta_{2}, \theta_{3}\right)$ with $\left|\theta_{i}\right| \leq 1$, and

$$
\begin{gathered}
M_{1}^{T}=\left[\begin{array}{ll}
1 & 0 \\
1 & 0 \\
1 & 0
\end{array}\right], N_{11}=\left[\begin{array}{cc}
a_{11} & 0 \\
0 & a_{12} \\
0 & 0
\end{array}\right], N_{12}=\left[\begin{array}{c}
0 \\
0 \\
b_{1}
\end{array}\right], N_{21}=\left[\begin{array}{ll}
a_{21} & 0 \\
0 & a_{22} \\
0 & 0
\end{array}\right], N_{22}=\left[\begin{array}{c}
0 \\
0 \\
1
\end{array}\right], \\
M_{2}^{T}=\left[\begin{array}{l}
0 \\
b_{2}
\end{array}\right],
\end{gathered}
$$

in which parameter $a_{11}, a_{12}, a_{21}, a_{22}, b_{1}$, and $b_{2}$ are to be determined. The resulting fuzzy system is given by

$$
\begin{aligned}
\dot{x}= & \left(\alpha_{1}\left(A_{1}+\Delta A_{1}\right)+\alpha_{2}\left(A_{2}+\Delta A_{2}\right)\right) x \\
& +\left(\alpha_{1}\left(B_{1}+\Delta B_{1}\right)+\alpha_{2}\left(B_{2}+\Delta B_{2}\right)\right) u \\
& +\alpha_{1} D_{1}+\alpha_{2} D_{2},
\end{aligned}
$$

which is equivalent to

$$
\begin{aligned}
& \dot{x}_{1}=x_{2} \\
& \dot{x}_{2}=\left(\frac{g}{\frac{4 l}{3}-a m l} \alpha_{1}+\frac{2 g}{\pi\left(\frac{4 l}{3}-a m l \beta^{2}\right)} \alpha_{2}\right) x_{1} \\
&+\left(\frac{-\mu a}{\frac{4 l}{3}-a m l} \alpha_{1}+\frac{-\mu a}{\frac{4 l}{3}-a m l \beta^{2}} \alpha_{2}\right) u \\
&+\left(\alpha_{1} a_{11}+{ }_{2} a_{21}\right) \theta_{1} x_{1}+\left(\alpha_{1} a_{12}+{ }_{2} a_{22}\right) \theta_{2} x_{2} \\
&+\left(\alpha_{1} b_{1}+\alpha_{2} b_{2}\right) \theta_{3} u+\alpha_{1} d_{1}+\alpha_{2} d_{2} .
\end{aligned}
$$

Leaving $\Delta A_{i}, \Delta B_{i}$, and $d_{i}$ out of account, the difference between the resulting fuzzy system (58) and the actual pendulum model (56) is

$$
\Delta f=f_{x_{1}} x_{1}+f_{x_{2}} x_{2}+f_{u} u,
$$

where

$$
\begin{aligned}
f_{x_{1}}= & \frac{g \sin \left(x_{1}\right)}{\left(\frac{4 l}{3}-a m l \cos ^{2}\left(x_{1}\right)\right) x_{1}} \\
& -\left(\frac{g}{\frac{4 l}{3}-a m l} \alpha_{1}+\frac{2 g}{\pi\left(\frac{4 l}{3}-a m l \beta^{2}\right)} \alpha_{2}\right)
\end{aligned}
$$

$$
\begin{aligned}
f_{x_{2}}= & \frac{-\frac{a m l x_{2} \sin \left(2 x_{1}\right)}{2}}{\frac{4 l}{3}-a m l \cos ^{2}\left(x_{1}\right)} \\
f_{u}= & \frac{-\mu a \cos \left(x_{1}\right)}{\frac{4 l}{3}-a m l \cos ^{2}\left(x_{1}\right)} \\
& +\frac{\mu a}{\frac{4 l}{3}-a m l} \alpha_{1}+\frac{\mu a}{\frac{4 l}{3}-a m l \beta^{2}} \alpha_{2} .
\end{aligned}
$$

Here, we constrain $x_{2} \leq 10$. If we set $a_{11}=a_{21}=a_{1}$, $a_{12}=a_{22}=a_{2}$ and $=b_{1}=b_{2}=b$,

then they can be obtained as follows.

$$
\begin{gathered}
a_{1}=\max _{x_{1} \in[-\pi / 2, \pi / 2]}\left|f_{x_{1}}\right|=7.9358 \\
a_{2}=\max _{x_{1} \in[-\pi / 2, \pi / 2], x_{2} \in[-10,10]}\left|f_{x_{2}}\right|=0.8124 \\
b=\max _{x_{1} \in[-\pi / 2, \pi / 2]}\left|f_{u}\right|=176.4706 .
\end{gathered}
$$

With the above parameters for $M_{i}, N_{i 1}$ and $N_{i 2}$, we are ready for solving LMIs (54), or (41). Here, we use the software package Robust Control Toolbox (MATLAB) to solve (54), and obtain the following results:

$$
\begin{gathered}
\mathcal{K}_{1}=\left[\begin{array}{ll}
3.6236 & 0.8430
\end{array}\right], \quad \mathcal{K}_{2}=[3.62360 .8430] \\
Q^{-1}=\left[\begin{array}{ll}
0.6094 & 0.1365 \\
0.1365 & 0.0332
\end{array}\right] \times 10^{-3}, \quad \lambda_{\min }=0.0150
\end{gathered}
$$

where $\mathcal{M}$ is chosen as an $8 \times 8$ identity matrix. On the other hand, in order to obtain the variable gain $\Phi_{i}$ in (44), it is necessary to solve the adaptive law in (45), the related parameters are chosen as $\beta_{1}=\beta_{2}=0.1$, $\gamma_{1}=\gamma_{2}=2.0, \delta=\frac{2 b-\bar{b}}{\hat{d}_{c}^{2} \lambda_{\max } \bar{b}}-c$ with $c=0.5$, where $\mathrm{c}$ is automatically replaced by $c / 10$ if $\delta<0$. In this simulation, the initial state is set: $x_{10}=87^{\circ}, x_{20}=0 \mathrm{rad} / \mathrm{s}$.

To make a comparison between the proposed fuzzy controller and the regular one when using T-S fuzzy model, the inverted pendulum first is controlled by $\mathcal{K}_{i}$, which means the fuzzy controller $K_{i}$ is only composed of $\mathcal{K}_{i}$ and without $\Phi_{i}$. Fig.2 shows the control results, where the solid line, and dashed lines are associated with $x_{1}$, and $x_{2}$, respectively. The oscillations around time $3.14=\pi$ and $6.28=2 \pi$ are caused by the $2 \pi$-periodic square wave disturbance. Clearly, the pendulum is not be controlled at all without $\Phi_{k}$ since $x_{1}$ exceeded $\pi / 2$, which is the upper limit in consideration of the actual pendulum. By contrast, the control performance conducted by $K_{i}=\mathcal{K}_{i}+\Phi_{i}$ depicted in Figs. $3-4$, where Fig. 3 shows the responses for the state; 
Fig.4, control input. This simulation shows that the proposed fuzzy controller is able to balance the pendulum with external disturbance in range of $\left[-87^{\circ}, 87^{\circ}\right]$.

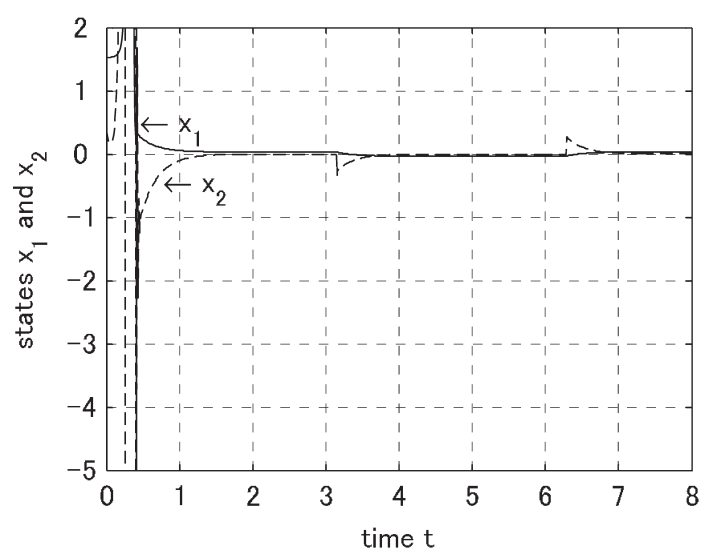

Fig.2 State $x_{1}$ and $x_{2}$ controlled by $K_{i}=\mathcal{K}_{i}$

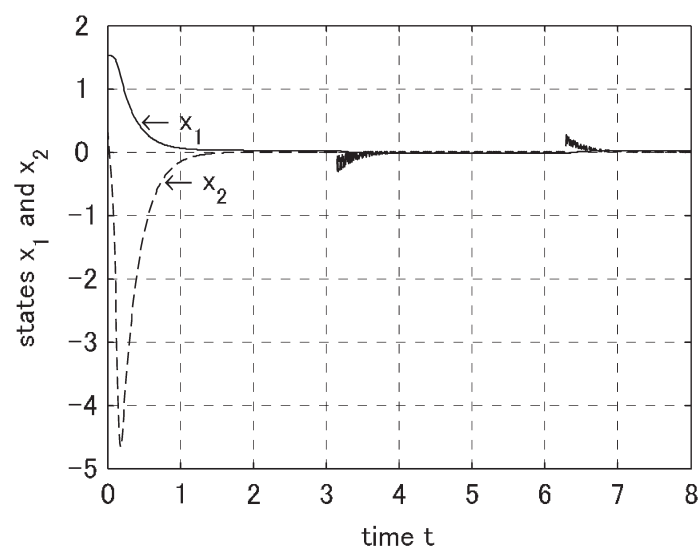

Fig.3 State $x_{1}$ and $x_{2}$ controlled by $K_{i}=\mathcal{K}_{i}+\Phi_{i}$

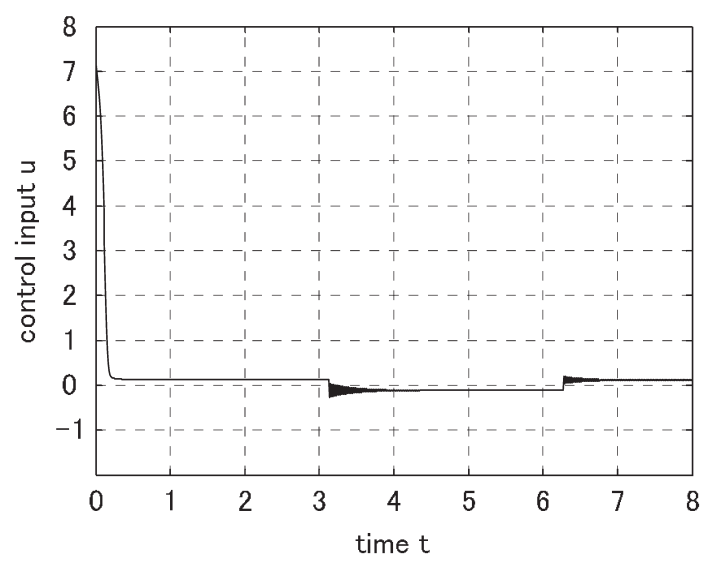

Fig.4 Control input composed of $K_{i}=\mathcal{K}_{i}+\Phi_{i}$

\section{Conclusions}

In this paper, in order to effectively deal with modeling error between the real system to be controlled and its T-S fuzzy model, an adaptive controller was proposed. Compared with the regular controller when using T-S fuzzy model, the proposed controller puts a variable part updated by an adaptive law besides the fixed one obtained by solving certain LMIs. The controller were applied to an inverted pendulum, and the simulation results confirmed the effectiveness.

\section{References}

[ 1 ] T. Takagi, and M. Sugeno, "Fuzzy identification of systems and its applications to modeling and control," IEEE Trans. Syst., Man, Cybern., vol.15, pp.116-132, 1985.

[ 2 ] M. Sugeno, and G.T. Kang, "Structure identification of fuzzy model," Fuzzy Sets Syst., vol.28, no.10, pp.1533, 1988.

[ 3 ] K. Tanaka, and M. Sugeno, "Stability analysis and design of fuzzy control systems," Fuzzy Sets Syst., vol.45, no.2, pp.135-156, 1992.

[ 4 ] K. Tanaka, T. Ikeda, and H.O. Wang, "Robust stabilization of a class of uncertain nonlinear systems via fuzzy control: Quadratic stabilizability, $H_{\infty}$ control theory, and linear matrix inequalities," IEEE Trans. Fuzzy Syst., vol.4, pp.1-13, 1996.

[ 5 ] K. Tanaka, and H.O. Wang, Fuzzy Control System Design and Analysis - A Linear Matrix Inequality Approach, Wiley, New York, 2001.

[6] Y.-Y Cao, and Z. Lin, "Robust stability analysis and fuzzy - scheduling control for nonlinear systems subject to actuator saturation," IEEE Trans. Fuzzy Syst., vol.11, no.1, pp.57-67, 2003.

[ 7 ] F. Zhang, Q.-G. Wang, and T. H. Lee, "Adaptive and robust controller design for uncertain nonlinear systems via fuzzy modeling approach," IEEE Trans. Syst., Man, and Cybern. vol.34, no.1, pp.166-178, 2004.

[ 8 ] B. Chen, X. Liu, S. Tong, and C. Lin,"Guaranteed cost control of T-S fuzzy systems with state and input delays,"Fuzzy Sets Syst., vol.158, pp.2251-2267, 2007.

[ 9 ] R.P. Khargonekar, I.R. Pertersen, and K. Zhou, "Robust stabilization of uncertain linear systems: quadratic stabilizability and $H_{\infty}$ control theory," IEEE Trans. Auto. Cont., no.35, no.3, pp.356-361, 1990.

[10] Z. Yi, and P.A. Heng, "Stability of fuzzy control systems with bounded uncertain delays," IEEE Trans. Fuzzy Syst., vol.10, no.1, pp.92-97, 2002.

(2008年 6 月11日 受付) (2009年 6 月 7 日 採録) 
[Contact Address]

Department of Management Information System Prefectural

University of Hiroshima

1-1-71 Ujina, Minami-ku, Hiroshima 734-8558, JAPAN

Hugang HAN

TEL : 082-251-9560

FAX : 082-251-9560

E-mail : hhan@pu-hiroshima.ac.jp

Hugang HAN [Member]

Hugang Han received the B.E. degree in control engineering from Northeast China Institute of Electric Power Engineering, Jilin, P. R. China in 1989, the M.S. degree in mechanical engineering from Yamagata University in 1992, and the Ph.D. degree in electric engineering from Kyushu Institute of Technology in 1997.

From 1992 to 1994 he was with the Department of Automation at Northeast China Institute of Electric Power Engineering. Since 1997, he has joined Prefectural University of Hiroshima where he is currently a Professor at the Department of Management and Information Sciences.

Dr. Han's main research interests include fuzzy control, adaptive control and complex system. 